\title{
The interplay between endogenous catecholamines and induced ventricular tachycardia during electrophysiologic testing
}

\begin{abstract}
Plaema epinephrine and norepinephrine concentrations were measured before, during, and shortly after induced ventricular tachycardia (VT) In 22 selected patients. Sustained, unimorphic VT was Induced by programmed ventricular stimulation and terminated after 45 to 384 seconds by overdrive pacing in all patients. In no patient did VT result in loss of consciousness. The baseline plaema catecholamine concentrations did not correlate with the baseline right ventricular effective refractory period, the cycle length of induced VT, or the number of extrastimuli required to induce VT. Induced VT was not associated with a significant increase In the mean plasma epinephrine concentration. In contrast, the plasma norepinephrine concentration Increased from a mean baseline level of $317 \pm 136 \mathrm{pg} / \mathrm{ml}$ (mean \pm standard deviation) to $418 \pm 220 \mathrm{pg} / \mathrm{ml}$ during VT $(p<0.01)$ and increased further to $569 \pm 387 \mathrm{pg} / \mathrm{ml}$ shortly after VT $(\rho<0.01)$. The plasma norepinephrine concentration shortly after VT correlated with the rate and duration of VT and with the magnitude of decrease in mean blood pressure during VT ( $p<0.05$ for each). In eight patients the same configuration of VT was induced on two sequential attempte; in five patients the same number of extrastimull were required for the second induction of VT as for the first, whereas in three patients fewer extrastimuli were required. Plasma cateholamine concentrations were not higher in patients requiring fewer extrastimuli to induce the second episode of VT, either shortly after the first episode of VT or shortly after the second eplsode of VT. In conclusion, plasma catecholamines do not influence baseline ventricular refractoriness, the cycle length of induced VT, or the VT induction technique. Induced VT, which does not require termination by direct-current countershock, is generally assoclated with little or no increase in plasma epinephrine and a variable increase in plasma norepinephrine concentration, depending on the severity and duration of hypotension during VT. The plasma catecholamine response to VT does not affect a second induction of VT. Therefore, endogenous catecholamines exert little influence on the results of electrophysiologic testing in patients with sustained VT which does not require termination by direct-current countershock. (Am Heart J 1987;113:227.)
\end{abstract}

Fred Morady, M.D., Jeffrey B. Halter, M.D., Lorenzo A. DiCarlo, Jr., M.D., Jeffrey M. Baerman, M.D., and Michael de Buitleir, M.B. Ann Arbor, Mich.

Isoproterenol administered during programmed ventricular stimulation shortens the ventricular effective refractory period and may facilitate the induction of ventricular tachycardia (VT)., ${ }^{1,2}$ This suggests the possibility that release of endogenous catecholamines may influence the ventricular effective refractory period and the technique necessary to induce VT during an electrophysiologic study.

From the Divisions of Cardiology and Geriatric Medicine, Department of Internal Medicine, University of Michigan Hospitals.

Received for publication May 23, 1986; accepted Aug. 11, 1986.

Reprint requests: Fred Morady, M.D., Division of Cardiology, University Hospitals, 1500 E. Medical Center Dr., UH-B1 F221-0022, Ann Arbor, MI 48109-0022.
However, no data are available ( $\mathrm{n}$ the relationship between endogenous catecholam ne levels and the ventricular effective refractory pe iod or the numbel of extrastimuli required to induc ! VT.

A prior study demonstrated the $t$ when VT requir. ing direct-current countershock is induced during programmed ventricular stimulat on, there is a several-fold increase in the plasme epinephrine and norepinephrine concentrations, ir association with 8 . 10 to $40 \mathrm{msec}$ decrease in the vintricular effective refractory period for up to 10 minutes after the countershock. ${ }^{3}$ However, no stu lies to date, have quantitated the plasma catechol umine response tc VT which does not require direct-current counter. shock. It is, therefore, unknown whether the cate. 
Table I. Characteristics of VT induced in 22 patients

\begin{tabular}{lcc}
\hline \multicolumn{1}{c}{ Characteristics } & Mean $\pm S D$ & Range \\
\hline $\begin{array}{l}\text { Bundle branch block configuration } \\
\quad \text { Right bundle branch block }\end{array}$ & 12 & - \\
$\quad$ Left bundle branch block & 10 & - \\
$\begin{array}{l}\text { Cycle length (msec) } \\
\text { Number of extrastimuli required }\end{array}$ & $328 \pm 62$ & $240-410$ \\
$\quad$ to induce VT & $4 \pm 0.6$ & $1-3$ \\
$\quad$ One & 14 & - \\
Two & 4 & - \\
Three & $135 \pm 92$ & - \\
$\begin{array}{l}\text { Duration of VT (sec) } \\
\text { Mean arterial pressure (mm Hg) } \\
\text { Baseline }\end{array}$ & $95 \pm 12$ & $73-120$ \\
At 30-45 seconds after onset & $76 \pm 17$ & $50-117$ \\
of VT & & \\
\hline
\end{tabular}

Abbreviations: $\mathrm{SD}=$ standard deviation; $\mathrm{VT}=$ ventricular tachycardia.

cholamine response to the first induction of VT may affect a second induction of VT.

This prospective study was designed to assess the interplay between endogenous catecholamines and VT induced during an electrophysiologic study. An attempt was made to answer the following questions: (1) Is there a relationship between the baseline plasma epinephrine or norepinephrine concentration and the baseline ventricular effective refractory period, the cycle length of induced VT, or the number of extrastimuli required to induce VT? (2) What determines the magnitude of rise in plasma catecholamines during VT not requiring countershock? (3) Does the plasma catecholamine response to the first induction of VT influence the next induction of VT?

\section{METHODS}

The subjects of this study were 22 patients who underwent an electrophysiologic study because of a history of sustained, unimorphic VT. Sustained, unimorphic VT was inducible by programmed ventricular stimulation in each patient, and none of the patients required direct-current countershock to terminate the VT. In each patient, the VT persisted until it was terminated by overdrive pacing. All 22 patients were men, and their mean age was $60 \pm 10$ years (mean \pm one standard deviation). All patients had undergone right and left heart catheterization and coronary angiography. Eighteen patients had coronary artery disease (with a history of myocardial infarction in 16), one patient had dilated cardiomyopathy, one patient had arrhythmogenic right ventricular dysplasia, and two patients had no evidence of structural heart disease. Eight patients had congestive heart failure, with a mean left ventricular ejection fraction, by contrast or radionuclide ventriculography, of $0.26 \pm 0.03$. The mean left ventricular ejection fraction in the 14 patients without congestive heart failure was $0.48 \pm 0.10$.

Electrophysiologic study protocol. Electrophysiologic studies were performed in the fasting, unsedated state after informed consent had been obtained. All antiarrhythmic drugs were discontinued at least five half-lives before the study. Electrode catheters were inserted percutaneously in a femoral vein and positioned against the right ventricular apex and either the right ventricular septum or outflow tract. A short 5 French cannula was inserted into a femoral artery for continuous monitoring of the arterial pressure. Leads $\mathrm{V}_{1}, \mathrm{I}$, and III, the intracardiac electrograms, and the arterial pressure were displayed on an oscilloscope and recorded on an Electronics for Medicine VR-16 or Siemens-Elema Mingograf 7 recorder. Programmed ventricular stimulation was performed with a programmable stimulator (Bloom Associates, Ltd., Narbeth, Pa.). The pacing stimuli were 2 msec in duration and twice the diastolic threshold.

Programmed ventricular stimulation was performed with the use of basic drive trains of eight stimuli at cycle lengths of 600,500 , and $400 \mathrm{msec}$. Single and double extrastimuli were introduced at the right ventricular apex, then at the second right ventricular site, followed by triple extrastimuli at the same two sites. In each patient, sustained VT was terminated by right ventricular overdrive pacing at cycle lengths 20 to 50 msec shorter than the VT cycle length. If direct-current countershock was required to terminate VT, the patient was not included in this study.

Catecholamine determinations. Two baseline samples of blood were obtained 5 minutes apart from the femoral artery, 15 minutes after placement of the electrode catheters in the right ventricle. A blood sample was obtained between 30 and 45 seconds after the onset of VT, before any attempts to terminate the VT by pacing. After the sample was drawn, right ventricular overdrive pacing was initiated to terminate the VT. Another blood sample was drawn between 15 and 30 seconds after the termination of VT.

The relationship between plasma catecholamines and the reproducibility of the technique used to induce VT was assessed in eight patients in whom the same configuration of VT was induced on successive attempts. Immediately after the post-VT blood sample was drawn, programmed ventricular stimulation was repeated at the same right ventricular site and basic drive cycle length used to induce the first episode of VT. A blood sample was drawn approximately every minute until VT was reinduced. Plasma epinephrine and norepinephrine concentrations were measured by means of a single isotope enzymatic assay. ${ }^{4}$ All samples from a given patient were analyzed in the same assay.

Data analysis. A paired $t$ test demonstrated no significant difference between the two baseline epinephrine and norepinephrine concentrations, and therefore the average of the two baseline values was used in the statistical analyses. The plasma catecholamine concentrations before, during, and after VT were compared by analysis of variance with repeated measures. Correlation coefficients were determined by linear regression analysis. Student's $t$ test was used to compare plasma catecholamine concen- 


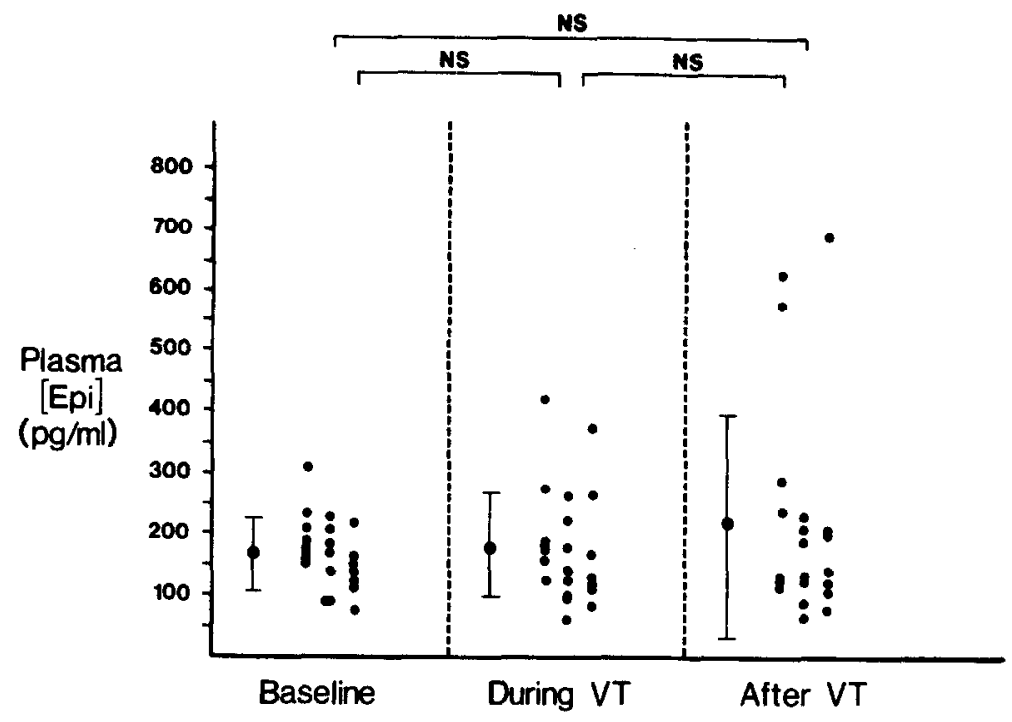

Fig. 1. Plasma epinephrine (Epi) concentrations before, during ( 30 to 45 seconds after onset), and shortly after an episode of induced VT. Individual data from 22 patients are shown, as well as mean \pm 1 SD.

trations in patients who required the same vs fewer extrastimuli to induce VT on a second occasion.

\section{RESULTS}

Relationship between baseline plasma catecholamines and refractoriness. The mean right ventricular effective refractory period determined at a basic drive cycle length of 500 msec was $239 \pm 16$ msec. There was not a significant correlation between either the baseline epinephrine or norepinephrine concentration and the right ventricular effective refractory period $(r=0.1$ and 0.2 , respectively; $p>0.3$ for both).

Characteristics of induced VT. Unimorphic VT was induced in each of the 22 patients. The characteristics of the induced VT are described in Table $\mathrm{I}$.

Effect of VT on plasma catecholamine concentrations. There were no significant differences between mean plasma epinephrine concentrations in the baseline state, $164 \pm 58 \mathrm{pg} / \mathrm{ml}$; during VT, $180 \pm 91$ $\mathrm{pg} / \mathrm{ml}$; and shortly after termination of the $\mathrm{VT}$, $219 \pm 180 \mathrm{pg} / \mathrm{ml}(p>0.05$; Fig. 1). However, in 4 of the 22 patients the plasma epinephrine concentration shortly after termination of VT exceeded the baseline concentration by more than $100 \mathrm{pg} / \mathrm{ml}$.

The mean plasma norepinephrine concentration in the baseline state was $317 \pm 136 \mathrm{pg} / \mathrm{ml}$. There was a significant increase in the mean plasma norepinephrine concentration to $418 \pm 220 \mathrm{pg} / \mathrm{ml}$ during VT $(p<0.01)$ and a further significant increase to $569 \pm 387 \mathrm{pg} / \mathrm{ml}$ shortly after termination of the VT ( $p<0.01$; Fig. 2). A progressive increase in the plasma norepinephrine concentration from the

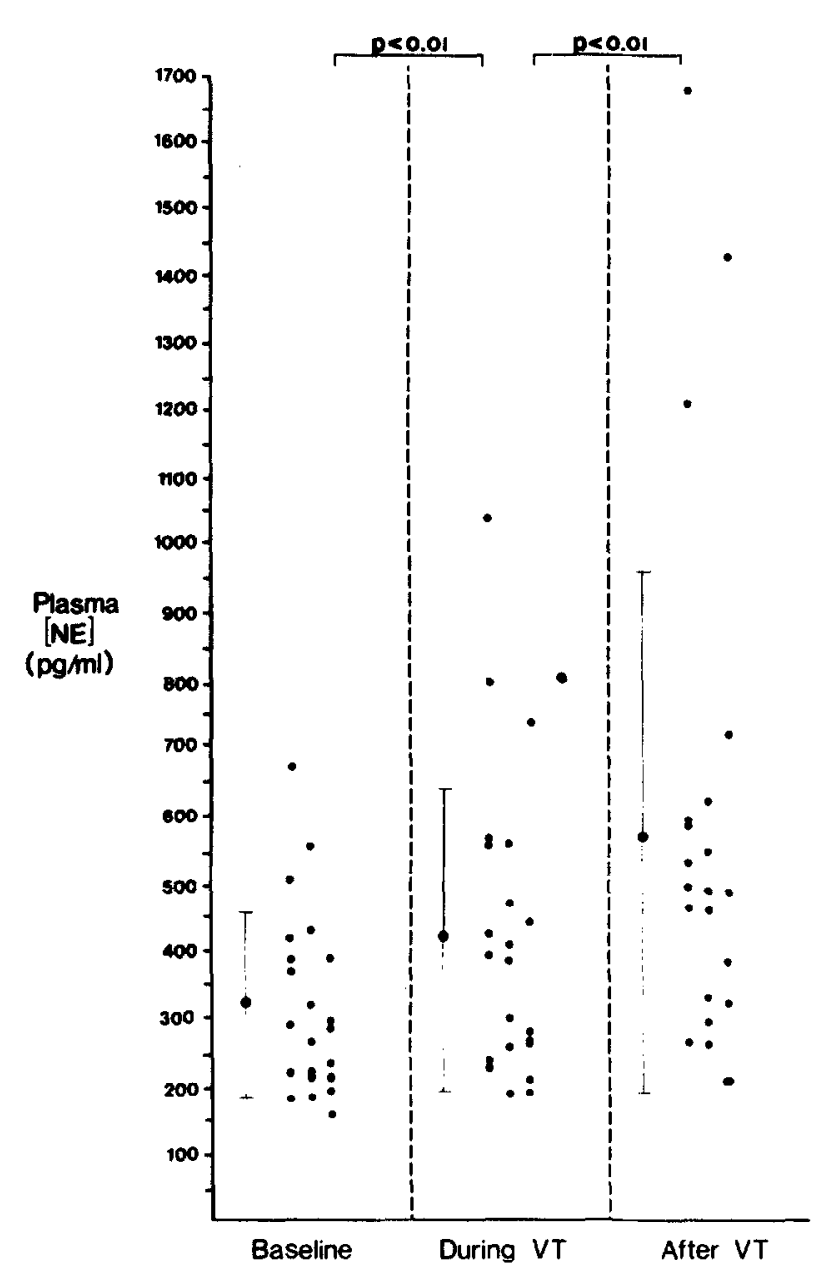

Fig. 2. Plasma norepinephrine (NE) concentrations before, during (30 to 45 seconds after onset), and shortly after an episode of induced VT. Format as in Fig. 1. 


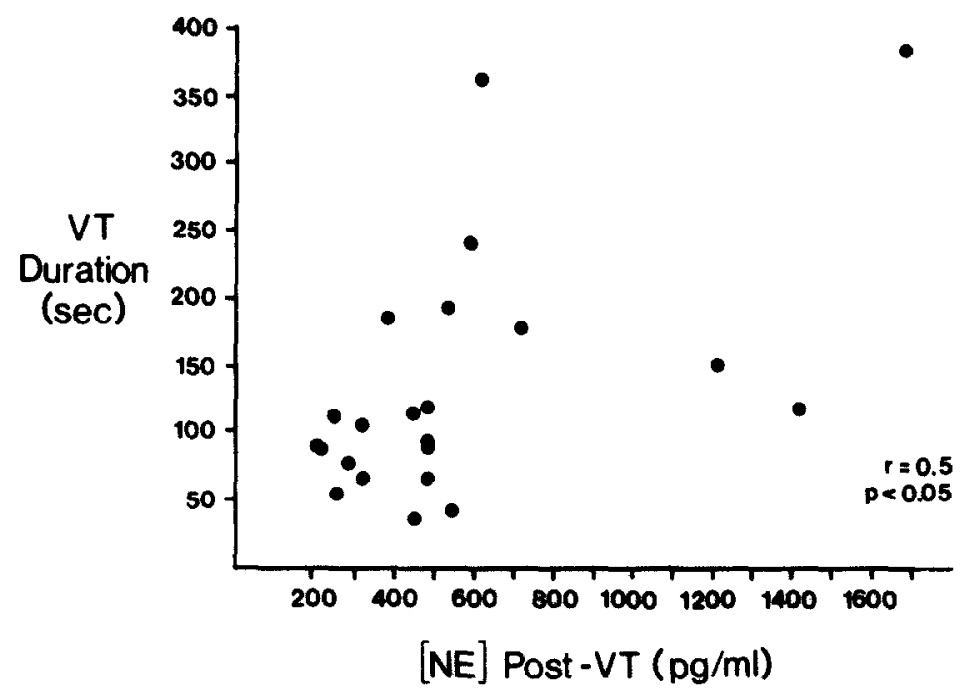

Fig. 3. Correlation between duration of induced VT and plasma norepinephrine concentration shortly after termination of VT in 22 patients.

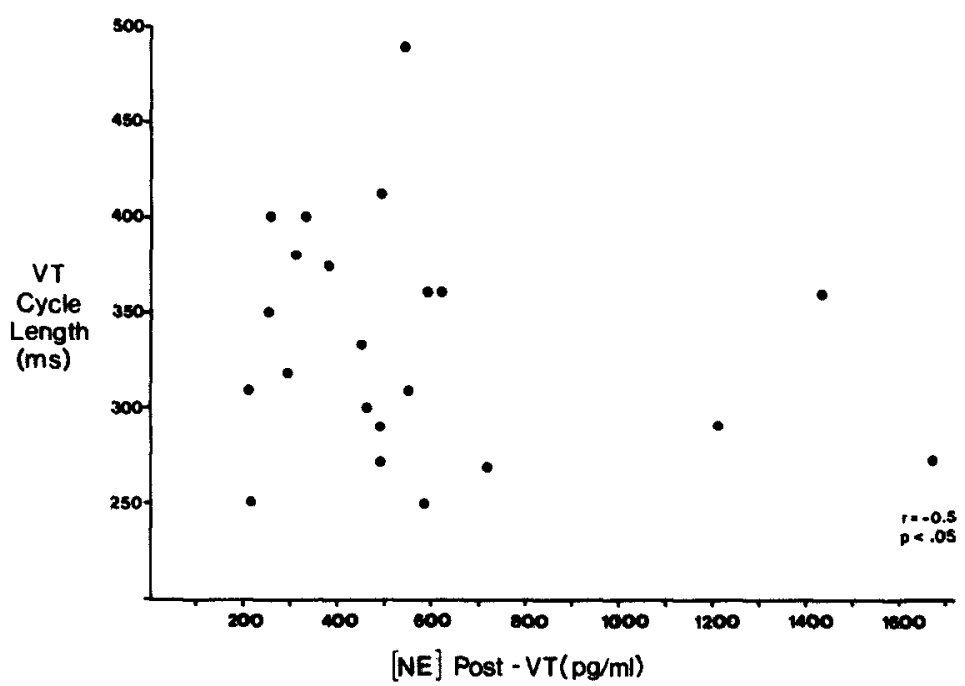

Fig. 4. Correlation between cycle length of induced VT and plasma norepinephrine concentration shortly after termination of VT.

baseline state to during VT to shortly after VT was observed in 15 of 22 patients.

Rolationship between baselline plasma catecholamines and Induced VT. There was not a significant correlation between either the baseline epinephrine or norepinephrine concentration and the cycle length of induced VT $(r=0.06$ and 0.01 , respectively; $p>0.8$ for both). Comparing the four patients in whom VT was induced by a single extrastimulus, the 14 in whom it was induced by two extrastimuli, and the four in whom it was induced by three extrastimuli, there were no significant difierences in the baseline epinephrine or norepinephrine concentra- tions between the three groups of patients $(p>0.05)$.

Plasma catecholemines shortly after termination of VT. The average duration of VT was $135 \pm 92$ seconds, with a range of 45 to 384 seconds. There was not a significant correlation between the duration of VT and the plasma epinephrine concentration shortly after termination of VT $(r=0.3$; $p=0.2)$. However, there was a significant correlation between the duration of VT and the plasma norepinephrine concentration shortly after termination of VT $(r=0.5, p<0.05$; Fig. 3$)$.

There was not a significant correlation between 

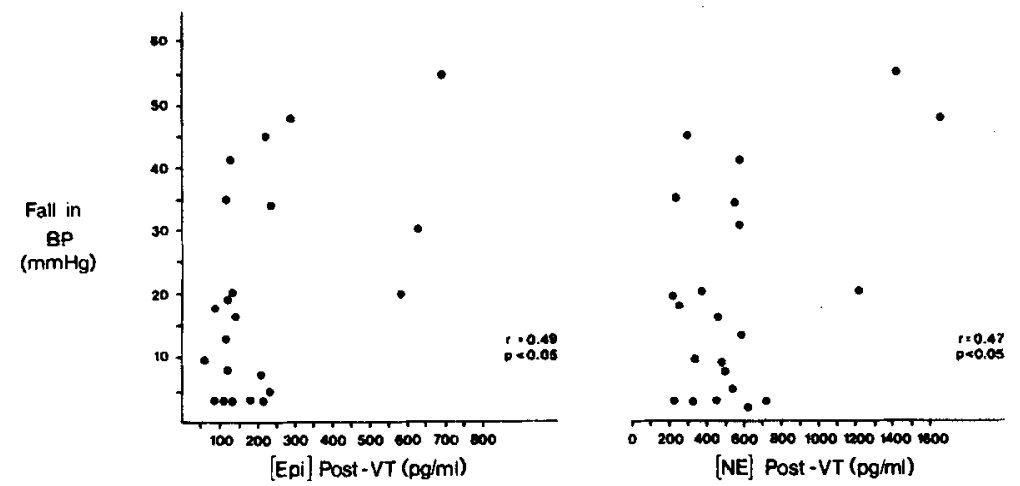

Fig. 5. Correlation between decrease in mean arterial blood pressure $(B P)$ during VT and plasma epinephrine and norepinephrine concentrations shortly after termination of VT.

the VT cycle length and the plasma epinephrine concentration shortly after termination of VT $(r=-0.4 ; p>0.05)$. However, there was a significant negative correlation between the cycle length of $\mathrm{VT}$ and the plasma norepinephrine concentration shortly after termination of VT $(r=-0.5, p<0.05$; Fig. 4).

There was a significant correlation between the change in mean blood pressure during VT and the plasma epinephrine and norepinephrine concentrations shortly after termination of VT $(r=0.49$ and 0.47 , respectively; $p<0.05$ for both; Fig. 5). There was also a significant correlation between the change in mean blood pressure during VT and the change from baseline in the plasma epinephrine and norepinephrine concentration shortly after termination of VT $(r=0.51 ; p<<0.05$, and $r=0.59 ; p<0.01$, respectively).

Plasma catecholamines in patients undergoing two inductions of VT. Plasma catecholamine concentrations were measured in serial fashion approximately every minute during programmed ventricular stimulation in eight patients in whom the same configuration of VT was induced on two occasions. In five patients the same number of extrastimuli was required for the first and second induction of VT, whereas in three patients a smaller number of extrastimuli was required for the second induction of VT as compared to the first induction.

The plasma epinephrine and norepinephrine concentrations in the baseline state, shortly after the first episode of VT, and shortly after the second induction of VT are listed in Table II. Of note is that there were no significant differences between the plasma epinephrine concentrations in patients who required either the same or fewer extrastimuli for the two inductions of VT. The plasma norepinephrine concentration shortly after the first episode of
Table II. Plasma catecholamines in patients undergoing two inductions of VT

\begin{tabular}{|c|c|c|c|}
\hline & \multicolumn{2}{|c|}{ No. of extrastimuli* } & \multirow[b]{2}{*}{$P$} \\
\hline & $\begin{array}{c}\text { Same } \\
(n=5)\end{array}$ & $\begin{array}{l}\text { Fewer } \\
(n=3)\end{array}$ & \\
\hline \multicolumn{4}{|c|}{ Plasma concentrations (pg/ml) } \\
\hline \multicolumn{4}{|c|}{ Baseline } \\
\hline Epinephrine & $165 \pm 15 \dagger$ & $137 \pm 25$ & NS \\
\hline Norepinephrine & $324 \pm 143$ & $208 \pm 46$ & NS \\
\hline \multicolumn{4}{|l|}{ Shortly after first VT } \\
\hline Epinephrine & $170 \pm 92$ & $157 \pm 59$ & NS \\
\hline Norepinephrine & $443 \pm 152$ & $233 \pm 51$ & $<0.05$ \\
\hline \multicolumn{4}{|c|}{ Shortly before second VT } \\
\hline Epinephrine & $154 \pm 90$ & $142 \pm 43$ & NS \\
\hline Norepinephrine & $362 \pm 150$ & $221 \pm 40$ & NS \\
\hline $\begin{array}{l}\text { Elapsed time between } \\
\text { first and second VT } \\
\text { (sec) }\end{array}$ & $163 \pm 62$ & $58 \pm 19$ & $<0.05$ \\
\hline
\end{tabular}

Abbreviations: $\mathrm{NS}$ = not significant; $\mathrm{VT}=$ ventricular tachycardia. *Number of extrastimuli required for second induction of VT compared to first induction.

†Mean \pm standard deviation.

VT was higher in patients who required the same number of extrastimuli for the two VT inductions than in patients who required fewer extrastimuli for the second VT induction $(p<0.05)$. However, there was not a significant difference in the plasma norepinephrine concentration between the two groups of patients shortly after the second episode of VT was induced.

The elapsed time between the end of the first episode of VT and the onset of the second VT was significantly shorter in patients who required fewer extrastimuli for the second VT induction than in patients who required the same number of extrastimuli for the two VT inductions ( $58 \pm 19$ seconds vs $163 \pm 62$ seconds, $p<0.05$; Table II). 
In two patients, the cycle length of the second induced VT was 20 to 30 msec shorter than the cycle length of the first induced VT, whereas in six patients the two episodes of VT had the same cycle length. Comparing these two subgroups there were no differences in the plasma epinephrine or norepinephrine concentration either shortly after the first episode of VT was terminated or shortly after the second episode of VT was induced.

\section{DISCUSSION}

The results of this study suggest that endogenous catecholamines exert little influence on the results of electrophysiologic testing in patients who have inducible unimorphic VT which does not require direct-current countershock to terminate. Whereas rapid VT that results in loss of consciousness and that is terminated by direct-current countershock is associated with a mean increase of fivefold in the plasma epinephrine concentration, ${ }^{3}$ sustained VT that is not associated with loss of consciousness and that can be terminated by right ventricular overdrive pacing is generally associated with little or no rise in the plasma epinephrine concentration, indicating little or no adrenal medullary response. Although the plasma norephinephrine concentration increased significantly in association with VT in the present study, the magnitude of the rise was, on the average, less than twofold. In patients in whom the same configuration of VT was induced on two occasions, a decrease in the number of extrastimuli required to induce VT was not related to an increase in the plasma catecholamine concentrations. Therefore, there appears to be little interplay between endogenous catechlamines and induced VT when direct-current countershock is not employed during an electrophysiology study.

In the present study, no correlation was demonstrated between the baseline right ventricular effective refractory period and the baseline plasma epinephrine or norepinephrine concentration. Therefore, it appears that the ventricular effective refractory period in the baseline state is not influenced by plasma catecholamine concentrations and that interpatient variation in ventricular refractoriness is not related to differences in the baseline degree of adrenergic activation.

The factors that determine the number of extrastimuli required to induce VT are unclear. Because isoproterenol has been demonstrated to facilitate the induction of VT by programmed ventricular stimulation, we considered the possibility that the number of extrastimuli required to induce VT may be related to the plasma epinephrine or norepinephrine concentration at the time of programmed stim- ulation. No difference was found in the mean plasma epinephrine or norepinephrine concentration between patients in whom VT was induced by single, double, or triple extrastimuli. Although the catecholamine concentrations were measured before instead of during programmed stimulation, a prior study demonstrated that programmed stimulation with a protocol identical to the one used in the present study does not affect plasma catecholamine concentrations. $^{3}$ Therefore, baseline plasma catecholamine levels do not appear to be a determinant of the number of extrastimuli required to induce VT.

Although there was not a significant increase in the mean epinephrine concentration in response to induced VT, the plasma epinephrine concentration increased to a level of greater than $550 \mathrm{pg} / \mathrm{ml}$ in 3 of 22 patients. There was also a heterogeneous response of the plasma norepinephrine concentration to induced VT. Whereas the mean plasma norepinephrine concentration increased by less than twofold, there was a twofold or greater increase in the plasma norepinephrine concentration in 6 of 22 patients. The plasma epinephrine concentration either during or shortly after VT did not correlate with the VT cycle length or duration, or with the blood pressure during the VT. However, there was a positive correlation between the norepinephrine concentration shortly after VT and both the duration of VT and the magnitude of the decrease in blood pressure during VT, and a negative correlation between the plasma norepinephrine concentration and the VT cycle length. These data suggest that the magnitude of increase in the plasma norepinephrine concentration in response to VT is determined, at least in part, by the severity and duration of hypotension during VT. It appears that the change in blood pressure may be more important than the absolute blood pressure in provoking a catecholamine response during VT.

In a prior study it was demonstrated that although unimorphic VT is usually reproducibly inducible, the number of extrastimuli required to induce the VT may vary in sequential induction attempts. ${ }^{5} \mathrm{~A}$ goal of this study was to determine whether changes in the number of extrastimuli required to induce VT may be related to increases in the plasma catecholamine concentrations that occur during the first episode of VT. In the eight patients in whom this was assessed, a decrease in the number of extrastimuli required to induce the second episode of VT could not be attributed to changes in the plasma catecholamine concentrations, since there was not a significant difference in the plasma epinephrine or norepinephrine concentrations before 
the induction of the first and second episodes of VT. Furthermore, the plasma norepinephrine concentration shortly after the first induction of VT was significantly higher among the five patients who required the same number of extrastimuli for the two inductions of VT than among the three patients in whom the second induction of VT required fewer extrastimuli than the first. These observations suggest that a change in the number of extrastimuli needed to induce VT cannot be attributed to adrenergic activation resulting from the first episode of VT.

In a prior study of the catecholamine response to VT associated with loss of consciousness and terminated by direct-current countershock, increases of several-fold in the plasma epinephrine and norepinephrine concentrations were observed at 1 minute after the countershock, but by 3 minutes after the countershock the plasma catecholamine concentrations were no longer significantly greater than baseline. ${ }^{3}$ Associated changes in ventricular refractoriness were greatest at 1 and 3 minutes after the countershock and did not persist for more than 10 minutes. These data indicate that even when elevations in plasma catecholamine concentrations are of large magnitude, the duration of the rise and associated electrophysiologic effects are shortlived. It may be possible that an episode of induced VT that is accompanied by marked and prolonged hypotension and that results in a several-fold increase in plasma catecholamines, could influence a second episode of VT induced within 1 to 3 minutes after termination of the first episode. However, in practice, there is usually a rest period of several minutes between episodes of induced VT. The relatively minor increase in plasma norepinephrine concentration that generally occurs in response to VT that is hemodynamically stable and that does not necessitate direct-current countershock is therefore highly unlikely to be of clinical importance.

Adrenergic activation would be expected to accelerate the rate of VT. Among the eight patients in whom the same configuration of VT was induced on two occasions in rapid succession, the cycle length of the second episode of VT was shorter than the cycle length of the first episode in only two patients. This provides additional evidence that the catecolamine response to hemodynamically significant VT is usually of little or no physiologic consequence.

A limitation of this study is that changes in the plasma norepinephrine concentration may not accurately reflect local change in norepinephrine at myocardial adrenergic synapses. Therefore, although no significant interplay between plasma catecholamine concentrations and induced VT was demonstrated in this study, interplay between local myocardial norepinephrine and induced VT cannot be ruled out. A second limitation is that changes in the number of extrastimuli needed to induce VT could be assessed in only eight patients. This was because in many patients a different configuration of VT was induced on a second attempt. A third limitation is that data on mean blood pressures during VT are only estimates. Precise measurement of the mean blood pressure during VT was not possible because of fluctuations over time and during periods of overdrive pacing.

In summary, the baseline state of adrenergic activity does not appear to be an important determinant of ventricular refractoriness, the number of extrastimuli required to induce VT, or the cycle length of induced VT. When sustained VT that is not accompanied by loss of consciousness and that does not require direct-current countershock is induced by programmed ventricular stimulation, there is generally little or no increase in the plasma epinephrine concentration and only a modest increase in the plasma norepinephrine concentration. The plasma catecholamine response is likely to be related to the magnitude and duration of the decrease in blood pressure that accompanies the VT. Adrenergic activation during induced VT does not appear to account for changes that may occur in the number of extrastimuli required to induce a subsequent episode of the same configuration of VT. The increases in plasma catecholamine concentration ihat may accompany an episode of induced VT not requiring countershock appear to be of litle or no physiologic consequence.

We gratefully acknowledge the technical assistance of Marla Smith, David Cahallan, Beverly Burgie, Joan Bergeron, and Linda Abbott; the computational assistance of Mr. Steven Schmaltz, MPH; and the secretarial assistance of Lisa Hackbarth.

\section{REFERENCES}

1. Reddy CP, Gettes LS. Use of isoproterenol as an aid to electric induction of chronic recurrent ventricular tachycardia. Am J Cardiol 1979;44:705.

2. Freedman RA, Swerdlow CD, Echt DS, Winkle RA, Soderholm-Difatte V, Mason JW. Facilitation of ventricular tachycardia induction by isoproterenol. Am J Cardiol 1984; 54:765.

3. Morady F, DiCarlo LA Jr, Halter JB, de Buitleir M, Krol RB, Baerman JM. The plasma catecholamine response to ventricular tachycardia induction and external countershock during electrophysiologic testing. J Am Coll Cardiol 1986;8:584.

4. Evans MI, Halter JB, Porte D Jr: Comparison of double- and single-isotope enzymatic derivative methods for measuring catecholamines in human plasma. Clin Chem 1978;24:567.

5. de Buitleir M, Morady F, DiCarlo LA Jr, Baerman JM, Krol RB. The immediate reproducibility of clinical and nonclinical forms of induced ventricular tachycardia. Am J Cardiol $1986 ; 58: 279$. 